CERN-TH/2002-310

hep-ph/0211010

November 2002

\title{
Impact of Bottom-Quark Measurements on our Knowledge of the Standard Model
}

\author{
Robert Fleischer \\ Theory Division, CERN, CH-1211 Geneva 23, Switzerland
}

\begin{abstract}
In this decade, $B$-decay experiments will allow stringent tests of the StandardModel description of $\mathrm{CP}$ violation. After a classification of the main strategies and a discussion of the most recent results on benchmark decay modes of $B^{ \pm}$ and $B_{d}$ mesons, we focus on the "El Dorado" for hadron colliders, the $B_{s^{-}}$ meson system, discussing the differences between the $B_{d}$ and $B_{s}$ systems, as well as prominent $B_{s}$ modes. First access to these decays is already provided by run II of the Tevatron. In the LHC era, it will be possible to fully exploit their physics potential, in particular at $\mathrm{LHCb}$ and $\mathrm{BTeV}$.
\end{abstract}

Invited talk at the 14th Topical Conference on Hadron Collider Physics, Karlsruhe, Germany, 29 September - 4 October 2002

To appear in the Proceedings 



\title{
Impact of Bottom-Quark Measurements on our Knowledge of the Standard Model
}

\author{
Robert Fleischer \\ Theory Division, CERN, CH-1211 Geneva 23, Switzerland
}

\section{Introduction}

In 1964 , the discovery of $\mathrm{CP}$ violation through $K_{\mathrm{L}} \rightarrow \pi^{+} \pi^{-}$decays came as a big surprise [1]. This particular kind of CP violation, which is described by the famous parameter $\varepsilon$, is referred to as "indirect" $\mathrm{CP}$ violation, as it is due to the fact that the $K_{\mathrm{L}}$ mass eigenstate is not an eigenstate of the $\mathrm{CP}$ operator with eigenvalue -1 , but receives a tiny admixture of the $\mathrm{CP}$ eigenstate with eigenvalue +1 . In 1999, also "direct" CP violation, i.e. CPviolating effects arising directly at the amplitude level, could be established in the neutral kaon system by the NA48 (CERN) [2] and KTeV (Fermilab) collaborations [3]. Unfortunately, the theoretical interpretation of the corresponding observable $\operatorname{Re}\left(\varepsilon^{\prime} / \varepsilon\right)$ is still affected by large hadronic uncertainties and does not provide a stringent test of the Standard-Model description of $\mathrm{CP}$ violation, unless significant theoretical progress concerning the relevant hadronic matrix elements can be made [4]- 6 .

One of the hot topics in this decade is the exploration of decays of $B$ mesons, allowing powerful tests of the CP-violating sector of the Standard Model (SM), and offering valuable insights into hadron dynamics [7]. At the moment, the stage is governed by the asymmetric $e^{+} e^{-} B$ factories operating at the $\Upsilon(4 S)$ resonance, with their detectors BaBar (SLAC) and Belle (KEK). In 2001, these experiments could establish $\mathrm{CP}$ violation in the $B$-meson system [8,9], which represents the start of a new era in the exploration of CP violation. Many interesting strategies can now be confronted with data [10]. In the near future, also run II of the Tevatron is expected to contribute significantly to this programme, providing - among other things - first access to $B_{s}$-meson decays [11]. In the LHC era, these decay modes can then be fully exploited [12], in particular at $\mathrm{LHCb}$ (CERN) and BTeV (Fermilab).

The focus of this overview is $\mathrm{CP}$ violation: in Section 2, we give an introduction to the SM description of this phenomenon, and classify the main strategies to explore it. In Section 3 , we shall then have a closer look at important benchmark modes of $B^{ \pm}$and $B_{d}$ mesons. The "El Dorado" for $B$-decay studies at hadron colliders, the $B_{s}$-meson system, is the subject of Section 4 , where we shall discuss the differences between the $B_{d}$ and $B_{s}$ systems, as well as prominent $B_{s}$ modes. In Section 5, we comment briefly on rare $B$ decays, before we summarize our conclusions and give an outlook in Section 6 . 


\section{CP Violation in $B$ Decays}

\subsection{Weak Decays}

The CP-violating effects we are dealing with in this paper originate from the charged-current interactions of the quarks, described by

$$
\mathcal{L}_{\text {int }}^{\mathrm{CC}}=-\frac{g_{2}}{\sqrt{2}}\left(\bar{u}_{\mathrm{L}}, \bar{c}_{\mathrm{L}}, \bar{t}_{\mathrm{L}}\right) \gamma^{\mu} \hat{V}_{\mathrm{CKM}}\left(\begin{array}{c}
d_{\mathrm{L}} \\
s_{\mathrm{L}} \\
b_{\mathrm{L}}
\end{array}\right) W_{\mu}^{\dagger}+\text { h.c. }
$$

where $g_{2}$ is $S U(2)_{\mathrm{L}}$ gauge coupling, the $W_{\mu}$ field corresponds to the charged $W$ bosons, and $\hat{V}_{\text {CKM }}$ denotes the Cabibbo-Kobayashi-Maskawa (CKM) matrix, connecting the electroweak eigenstates of the down, strange and bottom quarks with their mass eigenstates through a unitary transformation.

Since the CKM matrix elements $V_{U D}$ and $V_{U D}^{*}$ enter in $D \rightarrow U W^{-}$and the CP-conjugate process $\bar{D} \rightarrow \bar{U} W^{+}$, respectively, where $D \in\{d, s, b\}$ and $U \in\{u, c, t\}$, we observe that the phase structure of the CKM matrix is closely related to $\mathrm{CP}$ violation. It was pointed out by Kobayashi and Maskawa in 1973 that actually one complex phase is required - in addition to three generalized Euler angles - to parametrize the quark-mixing matrix in the case of three fermion generations, thereby allowing us to accommodate $\mathrm{CP}$ violation in the SM 13 .

The quark transitions caused by charged-current interactions exhibit an interesting hierarchy, which is made explicit in the Wolfenstein parametrization of the CKM matrix [14:

$$
\hat{V}_{\mathrm{CKM}}=\left(\begin{array}{ccc}
1-\frac{1}{2} \lambda^{2} & \lambda & A \lambda^{3}(\rho-i \eta) \\
-\lambda & 1-\frac{1}{2} \lambda^{2} & A \lambda^{2} \\
A \lambda^{3}(1-\rho-i \eta) & -A \lambda^{2} & 1
\end{array}\right)+\mathcal{O}\left(\lambda^{4}\right) .
$$

This parametrization corresponds to an expansion in powers of the small quantity $\lambda=0.22$, which can be fixed through semileptonic kaon decays. The other parameters are of order 1 , where $\eta$ leads to an imaginary part of the CKM matrix. The Wolfenstein parametrization is very useful for phenomenological applications, as we will see below.

\subsection{Unitarity Triangles}

The central targets for tests of the Kobayashi-Maskawa (KM) mechanism of $\mathrm{CP}$ violation are the unitarity triangles of the CKM matrix. As we have already noted, the CKM matrix is unitary, implying 6 orthogonality relations, which can be represented as 6 triangles in the complex plane [15], all having the same area [16]. However, using the Wolfenstein parametrization, it can 

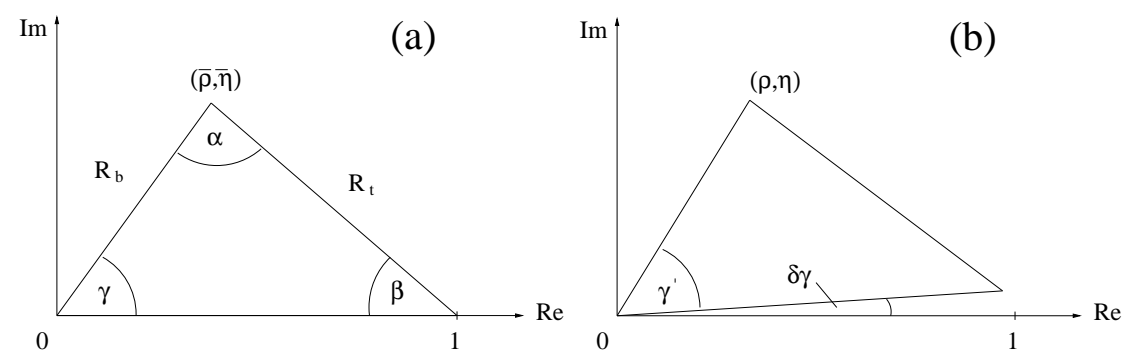

Fig. 1. The two non-squashed unitarity triangles of the CKM matrix: (a) and (b) correspond to the orthogonality relations (3) and (4), respectively.

be shown that only the following two relations describe triangles, where all three sides are of the same order of magnitude:

$$
\begin{aligned}
& V_{u d} V_{u b}^{*}+V_{c d} V_{c b}^{*}+V_{t d} V_{t b}^{*}=0 \\
& V_{u b}^{*} V_{t b}+V_{u s}^{*} V_{t s}+V_{u d}^{*} V_{t d}=0 .
\end{aligned}
$$

At leading order in $\lambda$, these relations agree with each other, and yield

$$
(\rho+i \eta) A \lambda^{3}+\left(-A \lambda^{3}\right)+(1-\rho-i \eta) A \lambda^{3}=0 .
$$

Consequently, they describe the same triangle, which is usually referred to as the unitarity triangle of the CKM matrix [16, 177. It is convenient to divide (5) by the overall normalization $A \lambda^{3}$. Then we obtain a triangle in the complex plane with a basis normalized to 1 , and an apex given by $(\rho, \eta)$.

In the future, the experimental accuracy will reach such an impressive level that we will have to distinguish between the unitarity triangles described by (3) and (四), which differ through $\mathcal{O}\left(\lambda^{2}\right)$ corrections. They are illustrated in Fig. 1. where $\bar{\rho}$ and $\bar{\eta}$ are related to $\rho$ and $\eta$ through [18]

$$
\bar{\rho} \equiv\left(1-\lambda^{2} / 2\right) \rho, \quad \bar{\eta} \equiv\left(1-\lambda^{2} / 2\right) \eta,
$$

and

$$
\delta \gamma \equiv \gamma-\gamma^{\prime}=\lambda^{2} \eta
$$

Whenever we refer to a unitarity triangle, we mean the one shown in Fig. 1 (a). To determine the allowed region in the $\bar{\rho}-\bar{\eta}$ plane, the "standard analysis" uses the following ingredients (for explicit expressions, see [19]):

- Exclusive and inclusive semileptonic $B$ decays caused by $b \rightarrow c \ell \bar{\nu}_{\ell}, u \ell \bar{\nu}_{\ell}$ quark-level transitions, fixing a circle of radius $R_{b}$ around $(0,0)$ [20].

- $B_{q}^{0}-\overline{B_{q}^{0}}$ mixing $(q \in\{d, s\})$, fixing a circle of radius $R_{t}$ around $(1,0)$.

- Indirect $\mathrm{CP}$ violation in the neutral kaon system, $\varepsilon$, fixing a hyperbola.

Many different strategies to deal with the corresponding theoretical and experimental uncertainties can be found in the literature. The most important 
ones are the simple scanning approach [5], the Gaussian approach [21], the BaBar 95\% scanning method [22], the Bayesian approach [23, and the nonBayesian statistical approach developed in [24]. A detailed discussion of these approaches is beyond the scope of this presentation. Let us here just give typical ranges for $\alpha, \beta$ and $\gamma$ that are implied by these strategies:

$$
70^{\circ} \lesssim \alpha \lesssim 130^{\circ}, \quad 20^{\circ} \lesssim \beta \lesssim 30^{\circ}, \quad 50^{\circ} \lesssim \gamma \lesssim 70^{\circ} .
$$

Direct determinations of these angles are provided by CP-violating effects in $B$ decays. The goal is now to overconstrain the unitarity triangle as much as possible through independent measurements of its sides and angles, with the hope to encounter discrepancies, which may shed light on new physics.

\subsection{Main Strategies}

The main rôle in the exploration of $\mathrm{CP}$ violation through $B$ decays is played by non-leptonic transitions, as CP-violating effects are due to interference effects, which arise in this decay class. In particular, interference between different decay topologies, i.e. tree and penguin contributions, may lead to direct $\mathrm{CP}$ violation. Unfortunately, the corresponding $\mathrm{CP}$ asymmetries are affected by hadronic matrix elements of local four-quark operators, which are hard to estimate and preclude a clean determination of weak phases. In order to solve this problem, we may employ one of the following approaches:

- The most obvious - but also most challenging - strategy we may follow is to try to calculate the relevant hadronic matrix elements $\left\langle\bar{f}\left|Q_{k}(\mu)\right| \bar{B}\right\rangle$. Interesting progress has recently been made in this direction through the development of the QCD factorization [25]-28], the perturbative hardscattering (PQCD) [29], and QCD light-cone sum-rule approaches [30].

- Another avenue we may follow is to search for fortunate cases where relations between decay amplitudes allow us to eliminate the hadronic matrix elements. Here we distinguish between exact relations, involving pure tree decays of the kind $B \rightarrow K D$ [31] 33] or $B_{c} \rightarrow D_{s} D$ [34], and relations, which follow from the flavour symmetries of strong interactions, involving $B_{(s)} \rightarrow \pi \pi, \pi K, K K$ decays 35 - 47.

- The third avenue we may follow to deal with the problems arising from hadronic matrix elements is to employ decays of neutral $B_{d}$ or $B_{s}$ mesons. Here we encounter a new kind of $\mathrm{CP}$ violation, which is due to interference effects between $B_{q}^{0-} \overline{B_{q}^{0}}(q \in\{d, s\})$ mixing and decay processes; it is referred to as "mixing-induced" $\mathrm{CP}$ violation. In the rate asymmetry

$$
\begin{aligned}
& \left.\frac{\Gamma\left(B_{q}^{0}(t) \rightarrow f\right)-\Gamma\left(\overline{B_{q}^{0}}(t) \rightarrow \bar{f}\right)}{\Gamma\left(B_{q}^{0}(t) \rightarrow f\right)+\Gamma\left(\overline{B_{q}^{0}}(t) \rightarrow \bar{f}\right)}\right|_{\Delta \Gamma_{q}=0} \\
& =\mathcal{A}_{\mathrm{CP}}^{\operatorname{dir}}\left(B_{q} \rightarrow f\right) \cos \left(\Delta M_{q} t\right)+\mathcal{A}_{\mathrm{CP}}^{\operatorname{mix}}\left(B_{q} \rightarrow f\right) \sin \left(\Delta M_{q} t\right),
\end{aligned}
$$


where $\Delta M_{q}$ and $\Delta \Gamma_{q}$ are the $B_{q}$ mass and decay widths differences, respectively, and $(C P)|f\rangle= \pm|f\rangle$, it is described by the coefficient of the $\sin \left(\Delta M_{q} t\right)$ term, whereas the one of $\cos \left(\Delta M_{q} t\right)$ measures direct CP violation. If the decay $B_{q} \rightarrow f$ is dominated by a single CKM amplitude, the corresponding hadronic matrix element cancels in $\mathcal{A}_{\mathrm{CP}}^{\operatorname{mix}}\left(B_{q} \rightarrow f\right)$. This observable is then simply given by $\pm \sin \left(\phi_{q}-\phi_{f}\right)$, where $\phi_{f}$ and $\phi_{q}$ are the weak $B_{q} \rightarrow f$ decay and $B_{q}^{0}-\overline{B_{q}^{0}}$ mixing phases, respectively [7].

\section{Benchmark Decay Modes of $B^{ \pm}$and $B_{d}$ Mesons}

\section{$3.1 \quad B \rightarrow \pi K$}

These decays, which originate from $\bar{b} \rightarrow \bar{d} d \bar{s}, \bar{u} u \bar{s}$ quark-level transitions, may receive contributions from penguin and tree topologies, where the latter bring the CKM angle $\gamma$ into the game. Interestingly, because of $\left|V_{u s} V_{u b}^{*} /\left(V_{t s} V_{t b}^{*}\right)\right| \approx$ $0.02, B \rightarrow \pi K$ modes are dominated by QCD penguins, despite their loop suppression. As far as electroweak (EW) penguins are concerned, they are expected to be negligible in $B_{d}^{0} \rightarrow \pi^{-} K^{+}, B^{+} \rightarrow \pi^{+} K^{0}$, as they contribute here only in colour-suppressed form. On the other hand, they are sizeable in $B^{+} \rightarrow \pi^{0} K^{+}$and $B_{d}^{0} \rightarrow \pi^{0} K^{0}$, i.e. of the same order of magnitude as the trees, since they contribute here also in colour-allowed form.

Through interference effects between tree and penguin contributions, we obtain sensitivity on $\gamma$. Relations between the $B \rightarrow \pi K$ amplitudes that are implied by the $S U(2)$ isospin flavour symmetry of strong interactions suggest the following combinations to determine this angle: the "mixed" $B^{ \pm} \rightarrow \pi^{ \pm} K$, $B_{d} \rightarrow \pi^{\mp} K^{ \pm}$system [36]- [39], the "charged" $B^{ \pm} \rightarrow \pi^{ \pm} K, B^{ \pm} \rightarrow \pi^{0} K^{ \pm}$ system [40]- 42], and the "neutral" $B_{d} \rightarrow \pi^{0} K, B_{d} \rightarrow \pi^{\mp} K^{ \pm}$system 42, 43].

All three $B \rightarrow \pi K$ systems can be described by the same set of formulae by just making straightforward replacements of variables 42 . Let us here, for simplicity, focus on the charged $B \rightarrow \pi K$ system. In order to determine $\gamma$ and strong parameters, we have to introduce appropriate CP-conserving and $\mathrm{CP}$-violating observables, which are given as follows:

$$
\left\{\begin{array}{l}
R_{\mathrm{c}} \\
A_{0}^{\mathrm{c}}
\end{array}\right\} \equiv 2\left[\frac{\mathrm{BR}\left(B^{+} \rightarrow \pi^{0} K^{+}\right) \pm \mathrm{BR}\left(B^{-} \rightarrow \pi^{0} K^{-}\right)}{\mathrm{BR}\left(B^{+} \rightarrow \pi^{+} K^{0}\right)+\mathrm{BR}\left(B^{-} \rightarrow \pi^{-} \overline{K^{0}}\right)}\right] .
$$

To parametrize these observables, we make use of the isospin relation mentioned above, and assume that certain rescattering effects are small, which is in accordance with the QCD factorization picture [25]- 27]. Anomalously large rescattering processes would be indicated by data on $B \rightarrow K K$ modes, which are already highly constrained by the $B$ factories, and could be taken into account through more elaborate strategies [39,41, 42]. The expressions for $R_{\mathrm{c}}$ and $A_{0}^{\mathrm{c}}$ thus obtained involve then - in addition to $\gamma$ - the parameters $r_{\mathrm{c}}$, $q$ and $\delta_{\mathrm{c}}$, which have the following physical interpretation: $r_{\mathrm{c}}$ measures, simply speaking, the ratio of tree to penguin topologies. It can be fixed through 

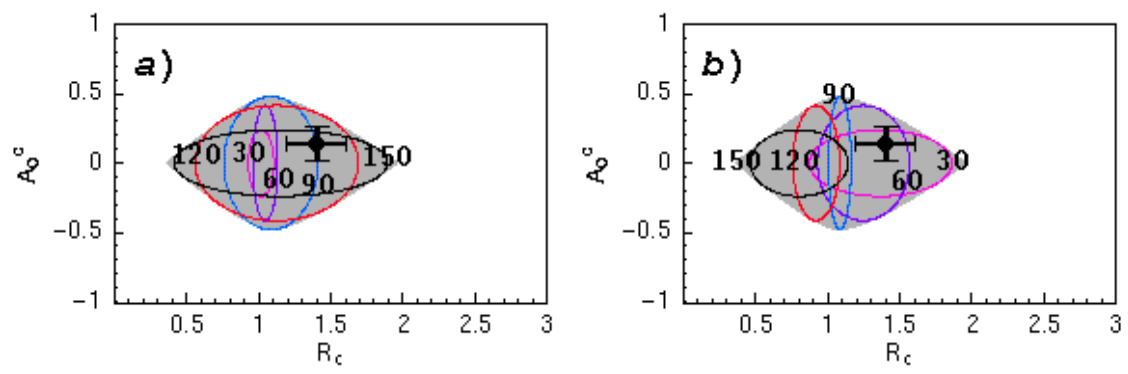

Fig. 2. The allowed regions in the $R_{\mathrm{c}}-A_{0}^{\mathrm{c}}$ plane for $q=0.68$ and $r_{\mathrm{c}}=0.24$. In (a) and (b), we show also the contours for fixed values of $\gamma$ and $\left|\delta_{\mathrm{c}}\right|$, respectively.

$S U(3)$ arguments and data on $B^{ \pm} \rightarrow \pi^{ \pm} \pi^{0}$ [35], yielding $r_{\mathrm{c}} \sim 0.2$. On the other hand, $q$ describes the ratio of $\mathrm{EW}$ penguin to tree contributions, and can be determined through $S U(3)$ arguments, yielding $q \sim 0.7$ 40]. Finally, $\delta_{\mathrm{c}}$ is the CP-conserving strong phase between trees and penguins.

Consequently, the two observables $R_{\mathrm{c}}$ and $A_{0}^{\mathrm{c}}$ depend on the two "unknowns" $\delta_{\mathrm{c}}$ and $\gamma$. If we vary them within their allowed ranges, i.e. $-180^{\circ} \leq$ $\delta_{\mathrm{c}} \leq+180^{\circ}$ and $0^{\circ} \leq \gamma \leq 180^{\circ}$, we obtain an allowed region in the $R_{\mathrm{c}^{-}}$ $A_{0}^{\mathrm{c}}$ plane 44,48 . Should the measured values of $R_{\mathrm{c}}$ and $A_{0}^{\mathrm{c}}$ lie outside this region, we would have an immediate signal for new physics. On the other hand, should the measurements fall into the allowed range, $\gamma$ and $\delta_{\mathrm{c}}$ could be extracted. In this case, $\gamma$ could be compared with the results of alternative strategies and with the values implied by the "standard analysis" of the unitarity triangle, whereas $\delta_{\mathrm{c}}$ provides valuable insights into hadron dynamics, thereby allowing tests of theoretical predictions.

In Fig. 2, we show the allowed regions in the $R_{\mathrm{c}}-A_{0}^{\mathrm{c}}$ plane for various parameter sets 48]. The crosses represent the averages of the present $B$-factory data. The contours in Figs. 2 (a) and (b) allow us to read off straightforwardly the preferred values for $\gamma$ and $\delta_{\mathrm{c}}$, respectively, from the measured observables. Interestingly, the present data seem to favour $\gamma \gtrsim 90^{\circ}$ (see also [49]), which would be in conflict with (8). Moreover, they point towards $\left|\delta_{\mathrm{c}}\right| \lesssim 90^{\circ}$; factorization predicts $\delta_{\mathrm{c}}$ to be close to $0^{\circ}$ 27. If future, more accurate data should really yield a value for $\gamma$ in the second quadrant, the discrepancy with (8) may be due to new-physics contributions to $B_{q}^{0}-\overline{B_{q}^{0}}$ mixing $(q \in\{d, s\})$ or the $B \rightarrow \pi K$ decay amplitudes. The allowed regions and contours in observable space of the neutral $B \rightarrow \pi K$ system look very similar to those shown in Fig. 2 [48]; for a recent update, see [50]. Unfortunately, the experimental situation in the neutral $B \rightarrow \pi K$ system is still rather unsatisfactory. As far as the mixed $B \rightarrow \pi K$ system is concerned, the present data fall well into the SM region in observable space, but do not yet allow us to draw further definite conclusions [48]. At present, the situation in the charged and neutral $B \rightarrow \pi K$ systems appears to be more exciting. Examples of the many other recent $B \rightarrow \pi K$ analyses can be found in [27,45, 46, 47, 51]. 


\section{$3.2 \quad B \rightarrow J / \psi K$}

The decay $B_{d}^{0} \rightarrow J / \psi K_{\mathrm{S}}$ is a transition into a CP-odd eigenstate, and originates from $\bar{b} \rightarrow \bar{c} c \bar{s}$ quark-level decays. Consequently, it receives contributions both from tree and from penguin topologies. Within the SM, we may write

$$
A\left(B_{d}^{0} \rightarrow J / \psi K_{\mathrm{S}}\right) \propto\left[1+\lambda^{2} a e^{i \theta} e^{i \gamma}\right],
$$

where the $\mathrm{CP}$-conserving hadronic parameter $a e^{i \theta}$ measures, sloppily speaking, the ratio of the penguin to tree contributions in $B_{d}^{0} \rightarrow J / \psi K_{\mathrm{S}}$ [52. Since this parameter enters in a doubly Cabibbo-suppressed way, we obtain to a very good approximation [53] (for a detailed discussion, see [7]):

$$
\mathcal{A}_{\mathrm{CP}}^{\mathrm{dir}}\left(B_{d} \rightarrow J / \psi K_{\mathrm{S}}\right)=0, \quad \mathcal{A}_{\mathrm{CP}}^{\mathrm{mix}}\left(B_{d} \rightarrow J / \psi K_{\mathrm{S}}\right)=-\sin \phi_{d},
$$

where $\phi_{d}$ denotes the CP-violating weak $B_{d}^{0}-\overline{B_{d}^{0}}$ mixing phase, which is given by $2 \beta$ in the SM. After important first steps by the OPAL, CDF and ALEPH collaborations, the $B_{d} \rightarrow J / \psi K_{\mathrm{S}}$ mode (and similar decays) led eventually, in 2001, to the observation of CP violation in the $B$ system [8.9]. The present status of $\sin 2 \beta$ is given as follows:

$$
\sin 2 \beta=\left\{\begin{array}{l}
0.741 \pm 0.067 \pm 0.033(\text { BaBar [54]) } \\
0.719 \pm 0.074 \pm 0.035 \text { (Belle [55]) }
\end{array}\right.
$$

yielding the world average 56 ]

$$
\sin 2 \beta=0.734 \pm 0.054,
$$

which agrees well with the results of the "standard analysis" of the unitarity triangle (8), implying $0.6 \lesssim \sin 2 \beta \lesssim 0.9$.

In the LHC era, the experimental accuracy of the measurement of $\sin 2 \beta$ may be increased by one order of magnitude [12]. In view of such a tremendous accuracy, it will then be important to obtain deeper insights into the theoretical uncertainties affecting (12), which are due to the penguin contributions described by $a e^{i \theta}$. A possibility to control them is provided by the $B_{s} \rightarrow J / \psi K_{\mathrm{S}}$ channel [52]. Moreover, also direct CP violation in $B \rightarrow J / \psi K$ modes allows us to probe such penguin effects [57,58]. So far, there are no experimental indications for non-vanishing $\mathrm{CP}$ asymmetries of this kind.

Although the agreement between (14) and the results of the CKM fits is striking, it should not be forgotten that new physics may nevertheless hide in $\mathcal{A}_{\mathrm{CP}}^{\text {mix }}\left(B_{d} \rightarrow J / \psi K_{\mathrm{S}}\right)$. The point is that the key quantity is actually $\phi_{d}$, which is fixed through $\sin \phi_{d}=0.734 \pm 0.054$ up to a twofold ambiguity,

$$
\phi_{d}=\left(47_{-4}^{+5}\right)^{\circ} \vee\left(133_{-5}^{+4}\right)^{\circ} \text {. }
$$

Here the former solution would be in perfect agreement with the range implied by the CKM fits, $40^{\circ} \lesssim \phi_{d} \lesssim 60^{\circ}$, whereas the latter would correspond to new 
physics. The two solutions can be distinguished through a measurement of the sign of $\cos \phi_{d}$ : in the case of $\cos \phi_{d}=+0.7>0$, we would conclude $\phi_{d}=47^{\circ}$, whereas $\cos \phi_{d}=-0.7<0$ would point towards $\phi_{d}=133^{\circ}$, i.e. new physics. There are several strategies on the market to resolve the twofold ambiguity in the extraction of $\phi_{d}$ [59. Unfortunately, they are rather challenging from a practical point of view. In the $B \rightarrow J / \psi K$ system, $\cos \phi_{d}$ can be extracted from the time-dependent angular distribution of the decay products of $B_{d} \rightarrow$ $J / \psi\left[\rightarrow \ell^{+} \ell^{-}\right] K^{*}\left[\rightarrow \pi^{0} K_{\mathrm{S}}\right]$, if the sign of a hadronic parameter $\cos \delta$ involving a strong phase $\delta$ is fixed through factorization 60, 61. This analysis is already in progress at the $B$ factories 62. For hadron colliders, the $B_{d} \rightarrow J / \psi \rho^{0}$, $B_{s} \rightarrow J / \psi \phi$ system is interesting to probe $\cos \phi_{d}$ [63].

The preferred mechanism for new physics to manifest itself in CP-violating effects in $B_{d} \rightarrow J / \psi K_{\mathrm{S}}$ is through $B_{d}^{0}-\overline{B_{d}^{0}}$ mixing, which arises in the SM from the famous box diagrams. However, new physics may also enter at the $B \rightarrow J / \psi K$ amplitude level. Employing estimates borrowed from effective field theory suggests that the effects are at most $\mathcal{O}(10 \%)$ for a generic newphysics scale $\Lambda_{\mathrm{NP}}$ in the $\mathrm{TeV}$ regime. In order to obtain the whole picture, a set of appropriate observables can be introduced, using $B_{d} \rightarrow J / \psi K_{\mathrm{S}}$ and its charged counterpart $B^{ \pm} \rightarrow J / \psi K^{ \pm}$[58]. So far, these observables do not indicate any deviation from the SM.

\section{$3.3 \quad B \rightarrow \phi K$}

Another important testing ground for the KM mechanism of $\mathrm{CP}$ violation is provided by $B \rightarrow \phi K$ decays, originating from $\bar{b} \rightarrow \bar{s} s \bar{s}$ quark-level transitions. These modes are governed by QCD penguins [64], but also EW penguins are sizeable [65]66]. Consequently, $B \rightarrow \phi K$ modes represent a sensitive probe for new physics. In the SM, we have the relations [57, 67, 68, 69]

$$
\begin{aligned}
& \mathcal{A}_{\mathrm{CP}}^{\mathrm{dir}}\left(B_{d} \rightarrow \phi K_{\mathrm{S}}\right)=0+\mathcal{O}\left(\lambda^{2}\right) \\
& \mathcal{A}_{\mathrm{CP}}^{\mathrm{mix}}\left(B_{d} \rightarrow \phi K_{\mathrm{S}}\right)=\mathcal{A}_{\mathrm{CP}}^{\mathrm{mix}}\left(B_{d} \rightarrow J / \psi K_{\mathrm{S}}\right)+\mathcal{O}\left(\lambda^{2}\right) .
\end{aligned}
$$

As in the case of the $B \rightarrow J / \psi K$ system, a combined analysis of $B_{d} \rightarrow \phi K_{\mathrm{S}}$, $B^{ \pm} \rightarrow \phi K^{ \pm}$modes should be performed in order to obtain the whole picture [69. There is also the possibility of an unfortunate case, where new physics cannot be distinguished from the SM, as discussed in [0,69].

In the autumn of 2002 , the experimental status can be summarized as

$$
\begin{aligned}
& \mathcal{A}_{\mathrm{CP}}^{\mathrm{dir}}\left(B_{d} \rightarrow \phi K_{\mathrm{S}}\right)= \begin{cases}\text { n.a. } & (\text { BaBar [70]) } \\
0.56 \pm 0.41 \pm 0.12 & (\text { Belle [71] })\end{cases} \\
& \mathcal{A}_{\mathrm{CP}}^{\mathrm{mix}}\left(B_{d} \rightarrow \phi K_{\mathrm{S}}\right)= \begin{cases}0.19_{-0.52}^{+0.50} \pm 0.09 & (\text { BaBar }[70]) \\
0.73 \pm 0.64 \pm 0.18 & \text { (Belle [71]). }\end{cases}
\end{aligned}
$$

Unfortunately, the experimental uncertainties are still very large. Because of $\mathcal{A}_{\mathrm{CP}}^{\operatorname{mix}}\left(B_{d} \rightarrow J / \psi K_{\mathrm{S}}\right)=-0.734 \pm 0.054$ (see (12) and (14)), there were already 
speculations about new-physics effects in $B_{d} \rightarrow \phi K_{\mathrm{S}}$ 72. In this context, it is interesting to note that there are more data available from Belle:

$$
\begin{gathered}
\mathcal{A}_{\mathrm{CP}}^{\mathrm{dir}}\left(B_{d} \rightarrow \eta^{\prime} K_{\mathrm{S}}\right)=-0.26 \pm 0.22 \pm 0.03 \\
\mathcal{A}_{\mathrm{CP}}^{\mathrm{mix}}\left(B_{d} \rightarrow \eta^{\prime} K_{\mathrm{S}}\right)=-0.76 \pm 0.36_{-0.05}^{+0.06} \\
\mathcal{A}_{\mathrm{CP}}^{\mathrm{dir}}\left(B_{d} \rightarrow K^{+} K^{-} K_{\mathrm{S}}\right)=0.42 \pm 0.36 \pm 0.09_{-0.03}^{+0.22} \\
\mathcal{A}_{\mathrm{CP}}^{\mathrm{mix}}\left(B_{d} \rightarrow K^{+} K^{-} K_{\mathrm{S}}\right)=-0.52 \pm 0.46 \pm 0.11_{-0.27}^{+0.03} .
\end{gathered}
$$

The corresponding modes are governed by the same quark-level transitions as $B_{d} \rightarrow \phi K_{\mathrm{S}}$. Consequently, it is probably too early to get too excited by the possibility of signals of new physics in $B_{d} \rightarrow \phi K_{\mathrm{S}}$ [56. However, the experimental situation should improve significantly in the future.

\section{$3.4 B \rightarrow \pi \pi$}

Another benchmark mode for the $B$ factories is $B_{d}^{0} \rightarrow \pi^{+} \pi^{-}$, which is a decay into a CP eigenstate with eigenvalue +1 , and originates from $\bar{b} \rightarrow \bar{u} u \bar{d}$ quark-level transitions. In the SM, we may write

$$
A\left(B_{d}^{0} \rightarrow \pi^{+} \pi^{-}\right) \propto\left[e^{i \gamma}-d e^{i \theta}\right],
$$

where the CP-conserving strong parameter $d e^{i \theta}$ measures, sloppily speaking, the ratio of penguin to tree contributions in $B_{d} \rightarrow \pi^{+} \pi^{-}$|73]. In contrast to the $B_{d}^{0} \rightarrow J / \psi K_{\mathrm{S}}$ amplitude (11), this parameter does not enter in (24) in a doubly Cabibbo-suppressed way, thereby leading to the well-known "penguin problem" in $B_{d} \rightarrow \pi^{+} \pi^{-}$. If we had negligible penguin contributions, i.e. $d=0$, the corresponding CP-violating observables were given as follows:

$$
\mathcal{A}_{\mathrm{CP}}^{\mathrm{dir}}\left(B_{d} \rightarrow \pi^{+} \pi^{-}\right)=0, \quad \mathcal{A}_{\mathrm{CP}}^{\mathrm{mix}}\left(B_{d} \rightarrow \pi^{+} \pi^{-}\right)=\sin (2 \beta+2 \gamma)=-\sin 2 \alpha,
$$

where we have also used the unitarity relation $2 \beta+2 \gamma=2 \pi-2 \alpha$. We observe that actually the phases $\phi_{d}=2 \beta$ and $\gamma$ enter directly in the $B_{d} \rightarrow \pi^{+} \pi^{-}$ observables, and not $\alpha$. Consequently, since $\phi_{d}$ can be fixed straightforwardly through $B_{d} \rightarrow J / \psi K_{\mathrm{S}}$, we may use $B_{d} \rightarrow \pi^{+} \pi^{-}$to probe $\gamma$ [48].

Measurements of the $B_{d} \rightarrow \pi^{+} \pi^{-}$CP asymmetries are already available:

$$
\begin{aligned}
& \mathcal{A}_{\mathrm{CP}}^{\mathrm{dir}}\left(B_{d} \rightarrow \pi^{+} \pi^{-}\right)= \begin{cases}-0.30 \pm 0.25 \pm 0.04 & (\text { BaBar [74]) } \\
-0.94_{-0.25}^{+0.31} \pm 0.09 & \text { (Belle [75]) }\end{cases} \\
& \mathcal{A}_{\mathrm{CP}}^{\mathrm{mix}}\left(B_{d} \rightarrow \pi^{+} \pi^{-}\right)= \begin{cases}-0.02 \pm 0.34 \pm 0.05 & (\text { BaBar [74]) } \\
1.21_{-0.38-0.16}^{+0.27+0.13} & \text { (Belle [75]). }\end{cases}
\end{aligned}
$$

Unfortunately, the BaBar and Belle results are not fully consistent with each other; the experimental picture will hopefully be clarified soon. Forming 
nevertheless the weighted averages of (26) and (27), using the rules of the Particle Data Group (PDG), yields

$$
\begin{aligned}
& \mathcal{A}_{\mathrm{CP}}^{\mathrm{dir}}\left(B_{d} \rightarrow \pi^{+} \pi^{-}\right)=-0.57 \pm 0.19(0.32) \\
& \mathcal{A}_{\mathrm{CP}}^{\text {mix }}\left(B_{d} \rightarrow \pi^{+} \pi^{-}\right)=0.57 \pm 0.25(0.61)
\end{aligned}
$$

where the errors in brackets are the ones increased by the PDG scaling-factor procedure [76. Direct $\mathrm{CP}$ violation at this level would require large penguin contributions with large CP-conserving strong phases. A significant impact of penguins on $B_{d} \rightarrow \pi^{+} \pi^{-}$is also indicated by data on $B \rightarrow \pi K, \pi \pi$ [48], as well as by theoretical considerations [27,51. Consequently, it is already evident that the penguin contributions to $B_{d} \rightarrow \pi^{+} \pi^{-}$cannot be neglected.

Many approaches to deal with the penguin problem in the extraction of weak phases from the CP-violating $B_{d} \rightarrow \pi^{+} \pi^{-}$observables were developed; for a selection, see [27,48,50,77]. In Subsection 4.3, we shall return to this issue by having a closer look at an approach using $B_{s} \rightarrow K^{+} K^{-}$[73].

\section{4 "El Dorado" for Hadron Colliders: $B_{s}$-Meson System}

\subsection{General Features}

At the $e^{+} e^{-} B$ factories operating at the $\Upsilon(4 S)$ resonance, no $B_{s}$ mesons are accessible, since $\Upsilon(4 S)$ states decay only to $B_{u, d}$-mesons, but not to $B_{s}$. On the other hand, the physics potential of the $B_{s}$ system is very promising for hadron machines, where plenty of $B_{s}$ mesons are produced. Consequently, $B_{s}$ physics is in some sense the "El Dorado" for $B$ experiments at hadron colliders. There are important differences between the $B_{d}$ and $B_{s}$ systems:

- Within the SM, the $B_{s}^{0}-\overline{B_{s}^{0}}$ mixing phase probes the tiny angle $\delta \gamma$ in the unitarity triangle shown in Fig. 1 (b), and is hence negligibly small:

$$
\phi_{s}=-2 \delta \gamma=-2 \lambda^{2} \eta=\mathcal{O}\left(-2^{\circ}\right)
$$

whereas $\phi_{d}=2 \beta=\mathcal{O}\left(50^{\circ}\right)$.

- A large $x_{s} \equiv \Delta M_{s} / \Gamma_{s}=\mathcal{O}(20)$ is expected in the SM, whereas $x_{d}=$ $0.775 \pm 0.012$. The present lower bound on $\Delta M_{s}$ is given as follows [78]:

$$
\Delta M_{s}>14.4 \mathrm{ps}^{-1} \text { (95\% C.L.). }
$$

- There may be a sizeable width difference $\Delta \Gamma_{s} / \Gamma_{s}=\mathcal{O}(-10 \%)$ between the mass eigenstates of the $B_{s}$ system, whereas $\Delta \Gamma_{d}$ is negligibly small 79. The present CDF and LEP results imply 78

$$
\left|\Delta \Gamma_{s}\right| / \Gamma_{s}<0.31 \text { (95\% C.L.). }
$$

Interesting applications of $\Delta \Gamma_{s}$ are extractions of weak phases from "untagged" $B_{s}$ data samples, where we do not distinguish between initially present $B_{s}^{0}$ or $\overline{B_{s}^{0}}$ mesons, as argued in [80]. 
Let us discuss the rôle of $\Delta M_{s}$ for the determination of the unitarity triangle in slightly more detail. The comparison of $\Delta M_{d}$ with $\Delta M_{s}$ allows an interesting determination of the side $R_{t}$ of the unitarity triangle. To this end, only a single $S U(3)$-breaking parameter $\xi$ is required, which measures $S U(3)$-breaking effects in non-perturbative mixing and decay parameters. It can be determined through lattice or QCD sum-rule calculations. The mass difference $\Delta M_{s}$ has not yet been measured. However, the lower bounds on $\Delta M_{s}$ can be converted into upper bounds on $R_{t}$ through [81]

$$
\left(R_{t}\right)_{\max }=0.83 \times \xi \times \sqrt{\frac{15.0 \mathrm{ps}^{-1}}{\left(\Delta M_{s}\right)_{\min }}},
$$

already excluding a large part in the $\bar{\rho}-\bar{\eta}$ plane, and implying in particular $\gamma<90^{\circ}$. In a recent paper 82, it is argued that $\xi$ may be significantly larger than the conventional range, $\xi=1.15 \pm 0.06 \rightarrow 1.32 \pm 0.10$. The excluded range in the $\bar{\rho}-\bar{\eta}$ plane would then be reduced, shifting the upper limit for $\gamma$ closer to $90^{\circ}$. Hopefully, the status of $\xi$ will be clarified soon. In the near future, run II of the Tevatron should provide a measurement of $\Delta M_{s}$, thereby constraining the unitarity triangle and $\gamma$ in a much more stringent way.

\subsection{Benchmark Decay Modes of $B_{s}$ Mesons}

An interesting class of $B_{s}$ decays is due to $b(\bar{b}) \rightarrow c \bar{u} s(\bar{s})$ quark-level transitions. Here we have to deal with pure tree decays, where both $B_{s}^{0}$ and $\overline{B_{s}^{0}}$ mesons may decay into the same final state $f$. The resulting interference effects between decay and mixing processes allow a theoretically clean extraction of $\phi_{s}+\gamma$ from

$$
\xi_{f}^{(s)} \times \xi_{\bar{f}}^{(s)}=e^{-2 i\left(\phi_{s}+\gamma\right)} .
$$

There are several well-known strategies on the market employing these features: we may consider the colour-allowed decays $B_{s} \rightarrow D_{s}^{ \pm} K^{\mp}$ [83], or the colour-suppressed modes $B_{s} \rightarrow D^{0} \phi$ [84]. In the case of $B_{s} \rightarrow D_{s}^{* \pm} K^{* \mp}$ or $B_{s} \rightarrow D^{* 0} \phi$, the observables of the corresponding angular distributions provide sufficient information to extract $\phi_{s}+\gamma$ from "untagged" analyses [85], requiring a sizeable $\Delta \Gamma_{s}$. A "tagged" strategy involving $B_{s} \rightarrow D_{s}^{* \pm} K^{* \mp}$ modes was proposed in 86. Recently, strategies making use of "CP-tagged" $B_{s}$ decays were proposed [87], which require a symmetric $e^{+} e^{-}$collider operated at the $\Upsilon(5 S)$ resonance. In this approach, initially present CP eigenstates $B_{s}^{\mathrm{CP}}$ are employed, which can be tagged through the fact that the $B_{s}^{0} / \overline{B_{s}^{0}}$ mixtures have anticorrelated $\mathrm{CP}$ eigenvalues at $\Upsilon(5 S)$. Here we may use the transitions $B_{s} \rightarrow D_{s}^{ \pm} K^{\mp}, D_{s}^{ \pm} K^{* \mp}, D_{s}^{* \pm} K^{* \mp}$. Let us note that there is also an interesting counterpart of (34) in the $B_{d}$ system 88], which employs $B_{d} \rightarrow D^{(*) \pm} \pi^{\mp}$ decays, and allows a determination of $\phi_{d}+\gamma$. 
The extraction of $\gamma$ from the phase $\phi_{s}+\gamma$ provided by the $B_{s}$ approaches sketched in the previous paragraph requires $\phi_{s}$ as an additional input, which is negligibly small in the Standard Model. Whereas it appears to be quite unlikely that the pure tree decays listed above are affected significantly by new physics, as they involve no flavour-changing neutral-current processes, this is not the case for the $B_{s}^{0}-\overline{B_{s}^{0}}$ mixing phase $\phi_{s}$. In order to probe this quantity, the decay $B_{s} \rightarrow J / \psi \phi$, which is the counterpart of $B_{d} \rightarrow J / \psi K_{\mathrm{S}}$, offers interesting strategies [61, 89$]$. In contrast to $B_{d} \rightarrow J / \psi K_{\mathrm{S}}$, the final state of $B_{s} \rightarrow J / \psi \phi$ is an admixture of different CP eigenstates. In order to disentangle them, we have to use the angular distribution of the $J / \psi \rightarrow \ell^{+} \ell^{-}$ and $\phi \rightarrow K^{+} K^{-}$decay products [90]. The corresponding observables are governed by 12

$$
\xi_{\psi \phi}^{(s)} \propto e^{-i \phi_{s}}\left[1-2 i \sin \gamma \times \mathcal{O}\left(10^{-3}\right)\right] .
$$

Since we have $\phi_{s}=\mathcal{O}\left(-2^{\circ}\right)$ in the SM, the extraction of $\phi_{s}$ from the $B_{s} \rightarrow J / \psi\left[\rightarrow \ell^{+} \ell^{-}\right] \phi\left[\rightarrow K^{+} K^{-}\right]$angular distribution may well be affected by hadronic uncertainties at the $10 \%$ level. These hadronic uncertainties, which may become an important issue in the LHC era [12], can be controlled through $B_{d} \rightarrow J / \psi \rho^{0}$, exhibiting some other interesting features [63]. Since $B_{s} \rightarrow J / \psi \phi$ shows small CP-violating effects in the SM because of (35), this mode represents a sensitive probe to search for new-physics contributions to $B_{s}^{0}-\overline{B_{s}^{0}}$ mixing [91]. For a detailed discussion of "smoking-gun" signals of a sizeable value of $\phi_{s}$, see 61. There, also methods to determine this phase unambiguously are proposed.

\subsection{The $B_{s} \rightarrow K^{+} K^{-}, B_{d} \rightarrow \pi^{+} \pi^{-}$System}

Since $B_{s} \rightarrow K^{+} K^{-}$and $B_{d} \rightarrow \pi^{+} \pi^{-}$are related to each other through an interchange of all down and strange quarks, the $U$-spin flavour symmetry of strong interactions allows us to express the four observables $\mathcal{A}_{\mathrm{CP}}^{\text {dir }}\left(B_{s} \rightarrow\right.$ $\left.K^{+} K^{-}\right), \mathcal{A}_{\mathrm{CP}}^{\mathrm{mix}}\left(B_{s} \rightarrow K^{+} K^{-}\right), \mathcal{A}_{\mathrm{CP}}^{\mathrm{dir}}\left(B_{d} \rightarrow \pi^{+} \pi^{-}\right), \mathcal{A}_{\mathrm{CP}}^{\mathrm{mix}}\left(B_{d} \rightarrow \pi^{+} \pi^{-}\right)$as functions of two hadronic penguin parameters $d$ and $\theta$, as well as $\gamma, \phi_{d}$ and $\phi_{s}$, which is negligibly small in the SM. Consequently, $d, \theta, \gamma, \phi_{d}$ can be determined. If $\phi_{d}$ is fixed through $B_{d} \rightarrow J / \psi K_{\mathrm{S}}$, the use of the $U$ spin symmetry in the extraction of $\gamma$ and the hadronic parameters can be minimized [73]. The approach has certain theoretical advantages, and is also very promising from an experimental point of view. At run II of the Tevatron and the LHC, one expects experimental accuracies for $\gamma$ of $\mathcal{O}\left(10^{\circ}\right)$ 11 and $\mathcal{O}\left(1^{\circ}\right)$ [12, respectively. For a collection of other $U$-spin strategies, see [52,63, 92].

Since $B_{s} \rightarrow K^{+} K^{-}$is not accessible at the $e^{+} e^{-} B$ factories operating at $\Upsilon(4 S)$, data are not yet available. However, $B_{s} \rightarrow K^{+} K^{-}$is related to $B_{d} \rightarrow \pi^{\mp} K^{ \pm}$through an interchange of spectator quarks. Consequently, we may approximately replace $B_{s} \rightarrow K^{+} K^{-}$by $B_{d} \rightarrow \pi^{\mp} K^{ \pm}$to deal with the 

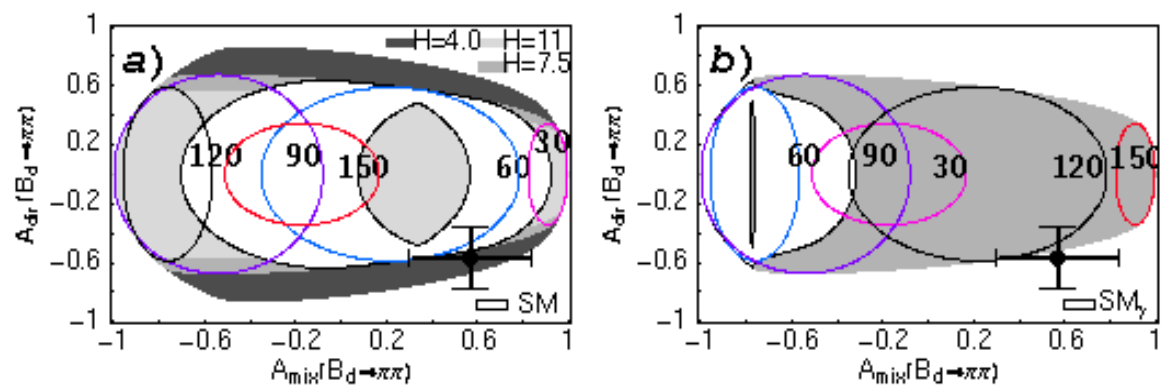

Fig. 3. Allowed regions in $B_{d} \rightarrow \pi^{+} \pi^{-}$observable space for (a) $\phi_{d}=47^{\circ}$ and various values of $H$, and (b) $\phi_{d}=133^{\circ}(H=7.5)$. The SM regions appear if we restrict $\gamma$ to (8). Contours representing fixed values of $\gamma$ are also included.

penguin problem in $B_{d} \rightarrow \pi^{+} \pi^{-}$93]. To this end, the quantity

$$
H=\frac{1}{\epsilon}\left(\frac{f_{K}}{f_{\pi}}\right)^{2}\left[\frac{\mathrm{BR}\left(B_{d} \rightarrow \pi^{+} \pi^{-}\right)}{\mathrm{BR}\left(B_{d} \rightarrow \pi^{\mp} K^{ \pm}\right)}\right]=\left\{\begin{array}{l}
7.3 \pm 2.9(\text { CLEO [94]) } \\
7.6 \pm 1.2(\text { BaBar [95]) } \\
7.1 \pm 1.9(\text { Belle 96]) }
\end{array}\right.
$$

is particularly useful, where $\epsilon \equiv \lambda^{2} /\left(1-\lambda^{2}\right)$. It allows us to eliminate the hadronic parameter $d$ in $\mathcal{A}_{\mathrm{CP}}^{\mathrm{dir}}\left(B_{d} \rightarrow \pi^{+} \pi^{-}\right)$and $\mathcal{A}_{\mathrm{CP}}^{\mathrm{mix}}\left(B_{d} \rightarrow \pi^{+} \pi^{-}\right)$, which then depend - for a given value of $\phi_{d}$ - only on $\gamma$ and the strong phase $\theta$. If we vary $\gamma$ and $\theta$ within their allowed ranges, we obtain an allowed region in the $\mathcal{A}_{\mathrm{CP}}^{\text {dir }}\left(B_{d} \rightarrow \pi^{+} \pi^{-}\right)-\mathcal{A}_{\mathrm{CP}}^{\mathrm{mix}}\left(B_{d} \rightarrow \pi^{+} \pi^{-}\right)$plane 48 , which is shown in Fig. 3. We observe that the experimental averages (28) and (29), represented by the crosses, overlap nicely with the SM region for $\phi_{d}=47^{\circ}$, and point towards $\gamma \sim 55^{\circ}$. In this case, not only $\gamma$ would be in accordance with the results of the CKM fits (8), but also $\phi_{d}$. On the other hand, for $\phi_{d}=133^{\circ}$, the experimental values favour $\gamma \sim 125^{\circ}$, and have essentially no overlap with the SM region. Since a value of $\phi_{d}=133^{\circ}$ would require CP-violating new-physics contributions to $B_{d}^{0}-\overline{B_{d}^{0}}$ mixing, also the $\gamma$ range in (8) may no longer hold in this case, as it relies on a Standard-Model interpretation of the experimental information on $B_{d, s}^{0}-\overline{B_{d, s}^{0}}$ mixing. In particular, also values for $\gamma$ larger than $90^{\circ}$ could then in principle be accommodated. As discussed in detail in [48], in order to put these statements on a more quantitative basis, we may use $H$ to calculate $\mathcal{A}_{\mathrm{CP}}^{\mathrm{dir}}\left(B_{d} \rightarrow \pi^{+} \pi^{-}\right)$for given values of $\mathcal{A}_{\mathrm{CP}}^{\mathrm{mix}}\left(B_{d} \rightarrow \pi^{+} \pi^{-}\right)$as a function of $\gamma$. Taking into account (28) and (29), we then obtain

$$
34^{\circ} \lesssim \gamma \lesssim 75^{\circ}\left(\phi_{d}=47^{\circ}\right), \quad 105^{\circ} \lesssim \gamma \lesssim 146^{\circ}\left(\phi_{d}=133^{\circ}\right) .
$$

In the future, the experimental uncertainties of the $B_{d} \rightarrow \pi^{+} \pi^{-}$observables will be reduced considerably, thereby providing significantly more stringent results for $\gamma$, as well as the hadronic parameters. 

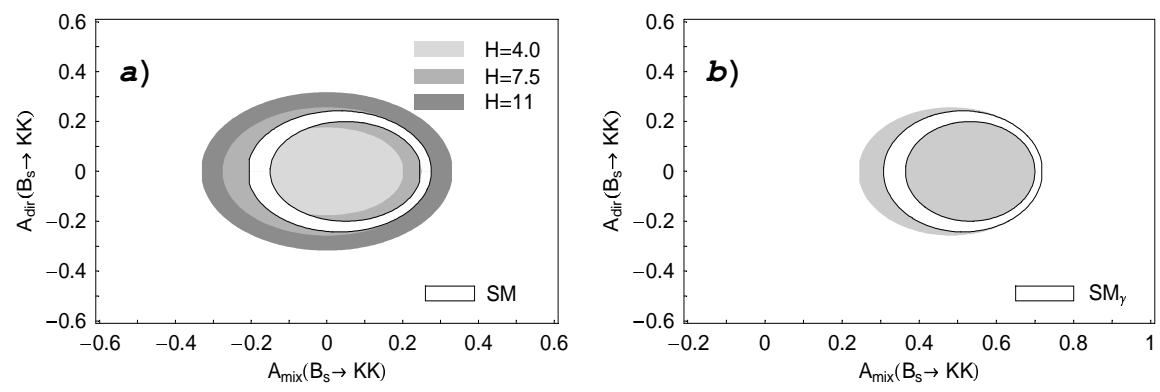

Fig. 4. Allowed regions in $B_{s} \rightarrow K^{+} K^{-}$observable space for (a) $\phi_{s}=0^{\circ}$ and various values of $H$, and (b) $\phi_{s}^{\mathrm{NP}}=30^{\circ}(H=7.5)$. The SM regions appear if $\gamma$ is restricted to $(8)$.

In analogy to the $B_{d} \rightarrow \pi^{+} \pi^{-}$analysis discussed above, we may also use $H$ to obtain an allowed region in the $\mathcal{A}_{\mathrm{CP}}^{\mathrm{mix}}\left(B_{s} \rightarrow K^{+} K^{-}\right)-\mathcal{A}_{\mathrm{CP}}^{\mathrm{dir}}\left(B_{s} \rightarrow K^{+} K^{-}\right)$ plane 48, as shown in Fig. 4. There, also the impact of a non-vanishing value of $\phi_{s}$, which may be due to new-physics contributions to $B_{s}^{0}-\overline{B_{s}^{0}}$ mixing, is illustrated. If we constrain $\gamma$ to (8), even more restricted regions appear. The allowed regions are remarkably stable with respect to variations of parameters characterizing $U$-spin-breaking effects [48, and represent a narrow target range for run II of the Tevatron and the experiments of the LHC era, in particular $\mathrm{LHCb}$ and $\mathrm{BTeV}$. These experiments will allow us to exploit the whole physics potential of the $B_{d} \rightarrow \pi^{+} \pi^{-}, B_{s} \rightarrow K^{+} K^{-}$system 73 .

\section{Comments on Rare $B$ Decays}

Let us finally comment briefly on rare $B$ decays, which occur at the one-loop level in the SM, and involve $\bar{b} \rightarrow \bar{s}$ or $\bar{b} \rightarrow \bar{d}$ flavour-changing neutral-current transitions. Prominent examples are $B \rightarrow K^{*} \gamma, B \rightarrow \rho \gamma, B \rightarrow K^{*} \mu^{+} \mu^{-}$and $B_{s, d} \rightarrow \mu^{+} \mu^{-}$. Within the SM, these modes exhibit small branching ratios at the $10^{-4}-10^{-10}$ level, and do not - apart from $B \rightarrow \rho \gamma$ - show sizeable $\mathrm{CP}$-violating effects, thereby representing important probes to search for new physics. For detailed discussions of the many interesting aspects of rare $B$ decays, the reader is referred to the overview articles listed in 97 .

\section{Conclusions and Outlook}

Decays of $B$ mesons represent a very exciting field of research. Thanks to the efforts of the BaBar and Belle collaborations, $\mathrm{CP}$ violation could recently be established in the $B$ system with the help of the "gold-plated" mode $B_{d} \rightarrow$ $J / \psi K_{\mathrm{S}}$, thereby opening a new era in the exploration of CP violation. The 
world average $\sin 2 \beta=0.734 \pm 0.054$ now agrees well with the SM expectation, but leaves a twofold solution $\phi_{d} \sim 47^{\circ} \vee 133^{\circ}$ for the $B_{d}^{0}-\overline{B_{d}^{0}}$ mixing phase itself. As the latter solution would point towards new physics, it is important to resolve this ambiguity directly.

The physics potential of $B$ experiments goes far beyond $B_{d} \rightarrow J / \psi K_{\mathrm{S}}$, allowing us now to confront many more CP strategies with data. Here the main goal is to overconstrain the unitarity triangle as much as possible, where $B \rightarrow \pi K, B \rightarrow \phi K$ and $B \rightarrow \pi \pi$ are important benchmark modes. Studies of $B$ decays at hadron colliders are an essential element of this programme, providing - among other things - access to the $B_{s}$-meson system. Already run II of the Tevatron is expected to yield interesting results on $B_{s}$ physics, and should discover $B_{s}^{0}-\overline{B_{s}^{0}}$ mixing soon, which is an important ingredient for the "standard" analysis of the unitarity triangle. Prominent $B_{s}$ decays are $B_{s} \rightarrow J / \psi \phi, B_{s} \rightarrow K^{+} K^{-}$and $B_{s} \rightarrow D_{s}^{ \pm} K^{\mp}$. Although we may obtain first valuable insights into these modes at the Tevatron, they can only be fully explored at the experiments of the LHC era, in particular LHCb and $\mathrm{BTeV}$.

\section{References}

1. J.H. Christenson et al., Phys. Rev. Lett. 13138 (1964)

2. V. Fanti et al. (NA48 Collaboration), Phys. Lett. B 465335 (1999);

J.R. Batley et al. (NA48 Collaboration), Phys. Lett. B 54497 (2002)

3. A. Alavi-Harati et al. (KTeV Collaboration), Phys. Rev. Lett. 8322 (1999); hep-ex/0208007

4. S. Bertolini, hep-ph/0206095

5. A.J. Buras, TUM-HEP-435-01 hep-ph/0109197

6. M. Ciuchini and G. Martinelli, Nucl. Phys. Proc. Suppl. B 9927 (2001)

7. For a recent review, see R. Fleischer, Phys. Rep. 370531 (2002)

8. B. Aubert et al. (BaBar Collaboration), Phys. Rev. Lett. 87091801 (2001)

9. K. Abe et al. (Belle Collaboration), Phys. Rev. Lett. 87091802 (2001)

10. The BaBar Physics Book, eds. P. Harrison and H.R. Quinn, SLAC-R-504 (1998)

11. K. Anikeev et al., FERMILAB-Pub-01/197 hep-ph/0201071

12. P. Ball et al., CERN-TH/2000-101 hep-ph/0003238]

13. M. Kobayashi and T. Maskawa, Prog. Theor. Phys. 49652 (1973)

14. L. Wolfenstein, Phys. Rev. Lett. 511945 (1983)

15. R. Aleksan, B. Kayser and D. London, Phys. Rev. Lett. 7318 (1994)

16. G.C. Branco and L. Lavoura, Phys. Lett. B 208123 (1988);

C. Jarlskog and R. Stora, Phys. Lett. B 208268 (1988)

17. L.L. Chau and W.Y. Keung, Phys. Rev. Lett. 531802 (1984)

18. A.J. Buras et al., Phys. Rev. D 503433 (1994)

19. A.J. Buras and R. Fleischer, Adv. Ser. Direct. High Energy Phys. 1565 (1998)

20. For a recent review, see Z. Ligeti, LBNL-49214 hep-ph/0112089

21. A. Ali and D. London, Eur. Phys. J. C $18665(2001)$

22. S. Plaszczynski and M.H. Schune, LAL-99-67 hep-ph/9911280; Y. Grossman, Y. Nir, S. Plaszczynski and M.H. Schune, Nucl. Phys. B 51169 (1998)

23. M. Ciuchini et al., JHEP 0107013 (2001)

24. A. Höcker et al., Eur. Phys. J. C 21225 (2001) 
25. M. Beneke et al., Phys. Rev. Lett. 831914 (1999)

26. M. Beneke et al., Nucl. Phys. B 591313 (2000)

27. M. Beneke et al., Nucl. Phys. B 606245 (2001)

28. C.W. Bauer, D. Pirjol and I.W. Stewart, Phys. Rev. Lett. 87201806 (2001); C.W. Bauer, B. Grinstein, D. Pirjol and I.W. Stewart, hep-ph/0208034

29. H.-n. Li and H.L. Yu, Phys. Rev. D 532480 (1996);

Y.Y. Keum, H.-n. Li and A.I. Sanda, Phys. Rev. D 63054008 (2001);

Y.Y. Keum and H.-n. Li, Phys. Rev. D 63074006 (2001)

30. A. Khodjamirian, Nucl. Phys. B 605558 (2001); B. Melić, hep-ph/0209265

31. M. Gronau and D. Wyler, Phys. Lett. B 265172 (1991)

32. I. Dunietz, Phys. Lett. B 27075 (1991).

33. D. Atwood, I. Dunietz and A. Soni, Phys. Rev. D 63036005 (2001); Phys. Rev. Lett. 783257 (1997)

34. R. Fleischer and D. Wyler, Phys. Rev. D 62057503 (2000)

35. M. Gronau, J.L. Rosner and D. London, Phys. Rev. Lett. 7321 (1994)

36. R. Fleischer, Phys. Lett. B 365399 (1996)

37. R. Fleischer and T. Mannel, Phys. Rev. D 572752 (1998)

38. M. Gronau and J.L. Rosner, Phys. Rev. D 576843 (1998)

39. R. Fleischer, Eur. Phys. J. C 6451 (1999); Phys. Lett. B 435221 (1998)

40. M. Neubert and J.L. Rosner, Phys. Lett. B 441403 (1998); Phys. Rev. Lett. 815076 (1998)

41. M. Neubert, JHEP 9902014 (1999)

42. A.J. Buras and R. Fleischer, Eur. Phys. J. C 1193 (1999)

43. A.J. Buras and R. Fleischer, Eur. Phys. J. C 1697 (2000)

44. R. Fleischer and J. Matias, Phys. Rev. D 61074004 (2000)

45. J. Matias, Phys. Lett. B 520131 (2001)

46. M. Bargiotti et al., Eur. Phys. J. C 24361 (2002)

47. M. Gronau and J.L. Rosner, Phys. Rev. D 65013004 [E: D 65 079901] (2002)

48. R. Fleischer and J. Matias, Phys. Rev. D 66054009 (2002)

49. M. Neubert, CLNS-02-1794 [hep-ph/0207327]

50. R. Fleischer, CERN-TH/2002-293 hep-ph/0210323

51. A.I. Sanda and K. Ukai, Prog. Theor. Phys. 107421 (2002); Y.-Y. Keum, DPNU-02-30 hep-ph/0209208

52. R. Fleischer, Eur. Phys. J. C 10299 (1999)

53. A.B. Carter and A.I. Sanda, Phys. Rev. Lett. 45952 (1980), Phys. Rev. D 23 1567 (1981); I.I. Bigi and A.I. Sanda, Nucl. Phys. B 19385 (1981)

54. B. Aubert et al. (BaBar Collaboration), hep-ex/0207042

55. K. Abe et al. (Belle Collaboration), hep-ex/0208025

56. Y. Nir, WIS-35-02-DPP hep-ph/0208080

57. R. Fleischer, Int. J. Mod. Phys. A 122459 (1997)

58. R. Fleischer and T. Mannel, Phys. Lett. B 506311 (2001)

59. Ya.I. Azimov, V.L. Rappoport and V.V. Sarantsev, Z. Phys. A 356437 (1997); Y. Grossman and H.R. Quinn, Phys. Rev. D 567259 (1997);

J. Charles et al., Phys. Lett. B 425375 (1998);

B. Kayser and D. London, Phys. Rev. D 61116012 (2000);

H.R. Quinn et al., Phys. Rev. Lett. 855284 (2000)

60. A.S. Dighe, I. Dunietz and R. Fleischer, Phys. Lett. B 433147 (1998)

61. I. Dunietz, R. Fleischer and U. Nierste, Phys. Rev. D 63114015 (2001)

62. R. Itoh, KEK-PREPRINT-2002-106 hep-ex/0210025 
63. R. Fleischer, Phys. Rev. D 60073008 (1999)

64. D. London and R.D. Peccei, Phys. Lett. B 223257 (1989);

N.G. Deshpande and J. Trampetic, Phys. Rev. D 41895 and 2926 (1990);

J.-M. Gérard and W.-S. Hou, Phys. Rev. D 432909 (1991)

65. R. Fleischer, Z. Phys. C 6281 (1994)

66. N.G. Deshpande and X.-G. He, Phys. Lett. B 336471 (1994)

67. Y. Grossman and M.P. Worah, Phys. Lett. B 395241 (1997)

68. D. London and A. Soni, Phys. Lett. B 40761 (1997)

69. R. Fleischer and T. Mannel, Phys. Lett. B 511240 (2001)

70. B. Aubert et al. (BaBar Collaboration). hep-ex/0207070

71. K. Abe et al. (Belle Collaboration), hep-ex/0207098

72. G. Hiller, SLAC-PUB-9326 hep-ph 0207356];

A. Datta, UDEM-GPP-TH-02-103 hep-ph/0208016;

M. Ciuchini and L. Silvestrini, hep-ph/0208087;

M. Raidal, CERN-TH/2002-182 hep-ph/0208091];

B. Dutta, C.S. Kim and S. Oh, hep-ph/0208226;

Jong-Phil Lee and Kang Young Lee, KIAS-P02054 hep-ph/0209290

73. R. Fleischer, Phys. Lett. B 459306 (1999)

74. B. Aubert et al. (BaBar Collaboration), hep-ex/0207055

75. K. Abe et al. (Belle Collaboration), Phys. Rev. Lett. 89071801 (2002)

76. K. Hagiwara et al. (Particle Data Group), Phys. Rev. D 66010001 (2002)

77. M. Gronau and D. London, Phys. Rev. Lett. 653381 (1990);

Y. Grossman and H.R. Quinn, Phys. Rev. D 58017504 (1998);

J. Charles, Phys. Rev. D 59054007 (1999);

D. London, N. Sinha and R. Sinha, Phys. Rev. D 63054015 (2001);

M. Gronau, D. London, N. Sinha and R. Sinha, Phys. Lett. B 514315 (2001);

M. Gronau and J.L. Rosner, Phys. Rev. D 65 093012; D 66053003 (2002)

78. Working group on $B$ oscillations, see http://lepbosc.web.cern.ch/LEPBOSC/

79. For a recent review, see M. Beneke and A. Lenz, J. Phys. G 271219 (2001)

80. I. Dunietz, Phys. Rev. D 523048 (1995);

R. Fleischer and I. Dunietz, Phys. Rev. D 55259 (1997)

81. A.J. Buras, TUM-HEP-259-96 hep-ph/9610461

82. A.S. Kronfeld and S.M. Ryan, Phys. Lett. B 54359 (2002)

83. R. Aleksan, I. Dunietz and B. Kayser, Z. Phys. C 54653 (1992)

84. M. Gronau and D. London, Phys. Lett. B 253483 (1991)

85. R. Fleischer and I. Dunietz, Phys. Lett. B 387361 (1996)

86. D. London, N. Sinha and R. Sinha, Phys. Rev. Lett. 851807 (2000)

87. A.F. Falk and A.A. Petrov, Phys. Rev. Lett. 85252 (2000)

88. I. Dunietz, Phys. Lett. B 427179 (1998)

89. A.S. Dighe, I. Dunietz and R. Fleischer, Eur. Phys. J. C 6647 (1999)

90. A.S. Dighe et al., Phys. Lett. B 369144 (1996)

91. Y. Nir and D.J. Silverman, Nucl. Phys. B 345301 (1990)

92. M. Gronau and J.L. Rosner, Phys. Lett. B 48271 (2000); M. Gronau, Phys. Lett. B 492297 (2000); P.Z. Skands, JHEP 0101008 (2001)

93. R. Fleischer, Eur. Phys. J. C 1687 (2000)

94. D. Cronin-Hennessy et al. (CLEO Coll.), Phys. Rev. Lett. 85515 (2000)

95. B. Aubert et al. (BaBar Collaboration), hep-ex/0207065 hep-ex/0206053

96. B.C. Casey et al. (Belle Collaboration), hep-ex/0207090

97. A. Ali, CERN-TH/2002-284 hep-ph/0210183;

A.J. Buras and M. Misiak, TUM-HEP-468-02 hep-ph/0207131;

G. Isidori, CERN-TH/2001-284 hep-ph/0110255] 